\title{
Morphological and Electrical Properties of Nanocellulose Compounds and Its Application on Capacitor Assembly
}

\author{
José Alfredo Hernández-Flores, ${ }^{1}$ Ana Beatriz Morales-Cepeda $\left(\mathbb{D},{ }^{2}\right.$ \\ Carlos Fernando Castro-Guerrero ${ }^{D},{ }^{3}$ Filemón Delgado-Arroyo, ${ }^{4}$ \\ Mario Roman Díaz-Guillén, ${ }^{4}$ Javier de la Cruz-Soto, ${ }^{3}$ Lorena Magallón-Cacho, ${ }^{3}$ \\ and Ulises León-Silva ${ }^{4}$ \\ ${ }^{1}$ Centro de Investigación en Ingeniería y Ciencias Aplicadas, Universidad Autónoma del Estado de Morelos, \\ Avenida Universidad 1001, Col. Chamilpa, Cuernavaca, Mor., C.P. 62209, Mexico \\ ${ }^{2}$ División de Estudios de Posgrado e Investigación, Instituto Tecnológico de Ciudad Madero, Juventino Rosas y Jesús Urueta s/n, \\ Col. Los Mangos, Cd. Madero, Tam., C.P. 89440, Mexico \\ ${ }^{3}$ CONACyT-Instituto Nacional de Electricidad y Energías Limpias, Reforma 113, Col. Palmira, Cuernavaca, Mor., \\ C.P. 62490, Mexico \\ ${ }^{4}$ Gerencia de Materiales y Procesos Químicos, Instituto Nacional de Electricidad y Energías Limpias, Reforma 113, Col. Palmira, \\ Cuernavaca, Mor., C.P. 62490, Mexico
}

Correspondence should be addressed to Carlos Fernando Castro-Guerrero; cfcastrog80@gmail.com

Received 31 December 2019; Revised 20 February 2020; Accepted 27 February 2020; Published 30 April 2020

Academic Editor: Zhonghua Peng

Copyright (C) 2020 José Alfredo Hernández-Flores et al. This is an open access article distributed under the Creative Commons Attribution License, which permits unrestricted use, distribution, and reproduction in any medium, provided the original work is properly cited.

\begin{abstract}
The rise for innovation in the electrical industry is strongly driven by development of new materials. Features of new materials are changing design paradigms for engineers. In this paper, the electrical properties of films of cellulose nanocrystals were measured. It was found that humidity affects the dielectric strength on the cellulose nanocrystals (CNCs). The dielectric strength was similar to the value of the industrial dielectric paper. The addition of plasticizer improved the flexibility of the material but lowered the dielectric strength. The films of CNC had an ordered arrangement, as suggested by the iridescence shown by them. The humidity content of the films was measured by thermogravimetric analysis. The CNC film was used for assembling a capacitor and compared to a capacitor assembled with dielectric paper.
\end{abstract}

\section{Introduction}

Cellulose nanocrystals are materials that are derived from cellulose [1]. Nanocellulose, as they are also called, result when the amorphous parts of cellulose are removed; after this, the crystalline parts remain on the material $[2,3]$. The higher crystallinity of CNC imparts advantages over normal cellulose. CNC has a higher storage and modulus than normal cellulose.

It also has higher chemical stability than normal cellulose, due to the higher crystallinity of CNC. According to KumarMishra et al. [4], experts are focusing the attention in appli- cations such as barrier for liquid and gaseous materials, biomedicine, and water purification. However, the appearance of low ecological footprint dielectric sheets based on nanocellulose is rising in cutting edge research [5], as an alternative to nanocomposite dielectrics [6]. There are reports on the mechanical and chemical properties of CNC, but there are very few dealing with the electrical properties of CNC. There are some papers reporting the use of CNC materials used for assembling capacitors [7] or for assembling batteries [8]. The interest of measuring the dielectric properties of CNCs resides on the fact that they are a promising material for electrical applications, due to its insulating 
properties and improved resistance, compared to paper [79]. Cellulose nanocrystals could be used for fabricating supercapacitors, batteries, and electric motors or in power transformers instead of dielectric paper. However, there are few papers dealing with the electrical properties and the dielectric behaviour of CNCs. Many products used in the electrical industry contain dielectric paper; this material degrades over time or absorbs humidity, causing it to lose its properties or fail, causing economic losses for both the supplier and the user. Products with improved properties would be an ideal choice for substituting them. CNCs are an economically accessible material with interesting properties that make them a material of potential applications in the energy sector.

In this report, we measured the dielectric properties of pure and compounded CNC films and an application of a capacitor device. We also made characterization with atomic force microscopy (AFM), thermogravimetric analysis (TGA), polarized optical microscopy (POM), and scanning electron microscopy (SEM). The characterization suggested that the films had an ordered phase of the cellulose nanocrystals. The dielectric properties suggest that CNCs may be a material of industrial interest in the energy sector, and they may substitute paper, as they have improved mechanical and chemical properties when compared to paper, while being less hygroscopic. The tests of the capacitor device suggest that the CNC films have potential for the fabrication of capacitors and that the $\mathrm{CNC}$ films are a good dielectric material.

\section{Materials and Methods}

Cellulose was extracted from cotton. Impurities were removed by boiling the samples in a $0.75 \mathrm{~N}$ solution for 3 hours; after this period of time, the $\mathrm{NaOH}$ solution was replaced by a new one, and the cellulose was boiled again for 3 hours [10]. After it, the cellulose was thoroughly rinsed with distilled water until the $\mathrm{pH}$ of filtrated water remained neutral. Then, the cellulose was washed with commercial $\mathrm{NaOCl}$ for $1.5 \mathrm{~h}$ at $45^{\circ} \mathrm{C}$. After it, the cellulose was washed with plenty of distilled water. Then, the cellulose was treated overnight with $\mathrm{HCl} 0.05 \mathrm{~N}$ in order to remove hemicellulose. After it, the cellulose was washed with distilled water and allowed to dry in an oven at $50^{\circ} \mathrm{C}$. Finally, the dried and purified cellulose was ground in a Wiley mill using a 20 -mesh sieve.

The cellulose powder was used to make CNC. One gram of cellulose was placed in a glass reactor with $17.5 \mathrm{~mL}$ of $\mathrm{H}_{2} \mathrm{SO}_{4} 64 \mathrm{wt} \%$ and allowed to react at $45^{\circ} \mathrm{C}$ for $45 \mathrm{~min}$. After the reaction, the solution was diluted by a factor of 10 with cold distilled water and allowed to settle; after it, the supernatant was discarded. Then, it was diluted again, and the process was repeated. The collected cellulose nanocrystals were dialyzed until the $\mathrm{pH}$ of the effluent remained constant or neutral. The purified nanocrystals were sonicated using a Sonics Vibra-Cell VCX 750 sonicator, at $65 \%$ output, until a colloidal suspension formed. The resulting solution was filtered using Whatman glass microfiber filters, for removing impurities introduced during sonication. The CNC suspension was dialyzed again for 2 days. Finally, the CNC suspen-

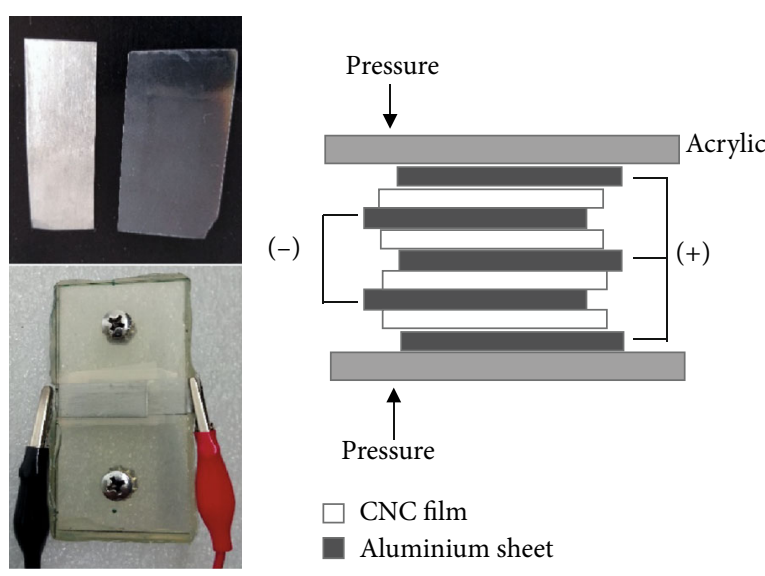

Figure 1: Al sheet, CNC film (dielectric separator), and image of the device and its architecture.

sion was spiked with toluene to avoid bacterial growth. The CNC suspension was placed in a chemical fridge. Aliquots of the CNC sample were taken and dried in an oven in order to determine gravimetrically the concentration of the CNC suspension. The measured concentration was $0.45 \mathrm{wt} \%$. CNC films were prepared with the CNC suspension. About $60 \mathrm{~mL}$ of CNC suspension was placed in a beaker; then, the suspension was sonicated in a Branson sonicating bath for 5 minutes, and then, it was degassed for 10 minutes to avoid bubble formation on the surface of the film, as the bubbles would produce inhomogenous surfaces that would facilitate the pass of the electrical current. Then, the CNC suspension was placed on a Petri dish and dried in an oven at $50^{\circ} \mathrm{C}$ until completely dried, and then, they were placed in a desiccator to avoid exposition to environmental moisture. To make flexible CNC films, a drop of glycerol was added to CNC suspensions, stirred for 1 hour at $40^{\circ} \mathrm{C}$, and then dried at $50^{\circ} \mathrm{C}$ in an oven.

Capacitor devices were tested using the CNC films. Aluminium (Al) and $\mathrm{CNC}$ sheets were cut (Figure 1) with the following dimensions: length $=30 \mathrm{~mm}(\mathrm{Al}, \mathrm{CNC})$ and width $=10 \mathrm{~mm}$ (Al) and $15 \mathrm{~mm}(\mathrm{CNC})$. Pretreatment of the $\mathrm{Al}$ sheets was carried out by polishing with two grades of emery papers (600 and 1200). The $\mathrm{Al}$ sheets were washed with distilled water, degreased with methyl alcohol, and dried at room temperature $\left(23^{\circ} \mathrm{C}\right)$. The thickness of the $\mathrm{Al}$ sheets was measured with a Mitutoyo digital micrometer. Three measurements were made to each sheet. The average value was measured and found to be $0.095 \mathrm{~mm}(95 \mu \mathrm{m})$. The capacitor was constructed as shown in Figure 1.

2.1. Characterization. The CNCs were characterized by Fourier-transform infrared (FTIR) spectroscopy and atomic force microscopy (AFM) and electrically. The FTIR was made with a Bruker spectrophotometer model Equinox 55 with an ATR accessory; the analysis was done in the IR range of $4000-750 \mathrm{~cm}^{-1}$ with a resolution of $2 \mathrm{~cm}^{-1}$, using the average of 64 scans. The AFM microscopy was done with a Nanosurf AFM, model Naio; the characterization was done in contact mode using a silicon probe. The images were analyzed using the software Gwyddion 2.47. The electrical 
TABLE 1: FTIR assignments of CNCs.

\begin{tabular}{lc}
\hline Group assignment & Frequency $\left(\mathrm{cm}^{-1}\right)$ \\
\hline$v(\mathrm{OH})$ & 3300 \\
$v_{\mathrm{s}}\left(\mathrm{CH}_{2}\right)$ & 2880 \\
$\delta_{\mathrm{s}}\left(\mathrm{CH}_{2}\right)$ & 1419 \\
$\delta_{\mathrm{s}}\left(\mathrm{CH}_{2}\right)$ & 1364 \\
$\delta(\mathrm{CH})_{\text {ring }}$ & 1319 \\
$\delta_{\mathrm{a}}(\mathrm{C}-\mathrm{O}-\mathrm{C})_{\text {bridge }}$ & 1020 \\
\hline
\end{tabular}

characterization was made by measuring the dielectric strength of the CNC films, using a Hipotronics, brand Hubbell, model 700 with gold cells, submerged in transformer oil that was previously dried. The dielectric strength characterization was made avoiding the formation of air bubbles. The thermal properties of the $\mathrm{CNC}$ film were measured with thermogravimetrical analysis (TGA), by using a TA Instruments thermogravimetric analyzer model TGA 2050; the analysis was made with a heating rate of $10^{\circ} \mathrm{C} / \mathrm{min}$ in an atmosphere of nitrogen. The films were observed with a Carl-Zeiss Polarized Optical Microscope, model AX10; the microscope has MOTIC software and camera.

Scanning electron microscopy was carried out on the $\mathrm{CNC}$ films to research its topographical characteristics and to confirm AFM results. The SEM was done with a CarlZeiss EVO MA 15. A small piece of the films was cut, and it was placed on carbon tape. The samples were observed at $1 \mathrm{kV}$ to avoid charging; the SEM characterization was performed in high vacuum without coating.

Electrochemical tests were performed at room temperature and controlled by a Gill AC potentiostat. Cyclic voltammetry was conducted over the potential range of 0 to $500 \mathrm{mV}, 1.5$ cycles, and scan rates of $10 \mathrm{mV} / \mathrm{s}, 20 \mathrm{mV} / \mathrm{s}$, and $50 \mathrm{mV} / \mathrm{s}$. Electrochemical impedance spectroscopy tests were carried out using an AC potential amplitude of $10 \mathrm{mV}$ and a frequency range of $0.1 \mathrm{~Hz}$ to $30 \mathrm{kHz}$ with 100 readings per decade.

\section{Results and Discussion}

The CNCs were characterized by several means. The FTIR characterization showed that the CNC samples have all the characteristic bands usually present on cellulose. These bands correspond to $\mathrm{OH}, \mathrm{C}-\mathrm{O}-\mathrm{C}$, and $\mathrm{CH}$ groups. The results are summarized in Table 1.

Table 1 shows that the $\mathrm{CNC}$ sample contains the main groups of cellulose $\left(\mathrm{CH}_{2}, \mathrm{CH}, \mathrm{OH}\right.$, and $\left.\mathrm{C}-\mathrm{O}-\mathrm{C}\right)$; this suggests that the dielectric behaviour of the sample should be similar to that of cellulose. The presence of the $\mathrm{OH}$ groups should also play an indirect role on the dielectric behaviour of the cellulose films, as they help to confirm that the CNCs are on the acid form. No neutralization or adding of an electrolyte was done on the CNC samples, so only the $\mathrm{OH}$ and sulfate groups should be on the sample. The $\mathrm{OH}$ and sulfate groups contribute to the hydrogen bonding of the CNC samples, as they have oxygen and hydrogen atoms. The hydrogen bonding contribute to the crystallinity, hydrophobicity, and mechanical properties of the samples, as after drying the
CNC suspensions on the acid form they cannot be dissolved again. The CNC films did not absorb water, nor did they dissolve again.

The morphology of the CNC films is shown in Figure 2. The CNC film (Figures 2(a) and 2(b)) shows a surface that has some features that generally are narrow and elongated. These features look like rods, but in a micrometric scale. The rest of the surface is even and mostly smooth. From the smooth surface, protrude the elongated features of the CNC film. The height of the hills observed on the sample was measured using the Gwyddion, and the result is $593.7 \mathrm{~nm} \pm 30.8 \mathrm{~nm}$. The length of the hills is $10.28 \mu \mathrm{m} \pm$ $0.82 \mu \mathrm{m}$, while the diameter is $1939 \mathrm{~nm} \pm 107.4 \mathrm{~nm}$. The dimensions of the nanocrystals forming the film were reported elsewhere in the literature [11]; they are $98.5 \mathrm{~nm} \pm 5 \mathrm{~nm}$ in length and $4.7 \mathrm{~nm} \pm 0.2 \mathrm{~nm}$ in diameter. Figures 2 (c) and 2(d) show the surface of a CNC sample with glycerol. The sample has a smooth surface, with some features looking like hills on the surface; they are formed due to the flexibility of the sample. There are also some small rodlike features on the sample. The morphology of the sample with glycerol lacks the features that are present on the CNC film imaged in Figures 2(a) and 2(b).

Figures $3(\mathrm{a})-3(\mathrm{~d})$ show the $2 \mathrm{D}$ and $3 \mathrm{D}$ images of a CNC film formed after drying a $5 \mathrm{wt} \% \mathrm{CNC}$ sample. The figure is interesting because there are features on the film pointing in a preferred direction, especially in Figures 3(c) and 3(d). In these last two figures, there are lines oriented in the same direction, mainly parallel between them. The lines seen on the sample are formed due to the ordering of the polymer chains of the materials; the lines are pointing to the orientation of rod axes of CNC [12]. The average separation between lines is $708.87 \mathrm{~nm} \pm 82.75 \mathrm{~nm}$. It has been reported that dried films formed by CNC suspensions keep the texture and orientation of the original suspension [13]. In this case, the sample was exposed to a magnetic field, so the Fréedericksz transition occurred and the $\mathrm{CNC}$ rods ordered in a straight line, instead of forming a chiral nematic phase. Figures 3(a) and 3(b) show features over the surface of the polymer film, which can be seen below the aforementioned features, near the centre and at the right side of Figure 3(a). These objects are agglomerations of CNCs; they are agglomerated over the CNC film. The agglomerates have a preferred orientation in Figures 3(a) and 3(b); the orientation is in a straight line, as the features observed in Figures 3(c) and 3(d). The dimensions of the agglomerates were measured with the Gwyddion; the length was $1144.98 \mathrm{~nm} \pm 49.08 \mathrm{~nm}$, while the diameter was $477.78 \mathrm{~nm} \pm 17.88 \mathrm{~nm}$. From Figure 3 , it can be inferred that the influence of the electrical field caused aligning and orientation of the nanocrystals. Hydroxypropyl cellulose (HPC, a cellulose derivative) compounds have shown in literature oriented lines when subjected to mechanical stress or when concentration reaches a critical concentration [12, 14]. HPC/polyacrylamide (HPC/PAAm) composites have been used for drug delivery using ibuprofen; they have shown ibuprofen crystals on the surface, similar to the crystals shown in Figures 3(a) and 3(b) [14]. Hydroxyethyl cellulose (HEC, a cellulose derivative)/polyacrylamide (HEC/PAAm) composites have also been used for drug delivery with 


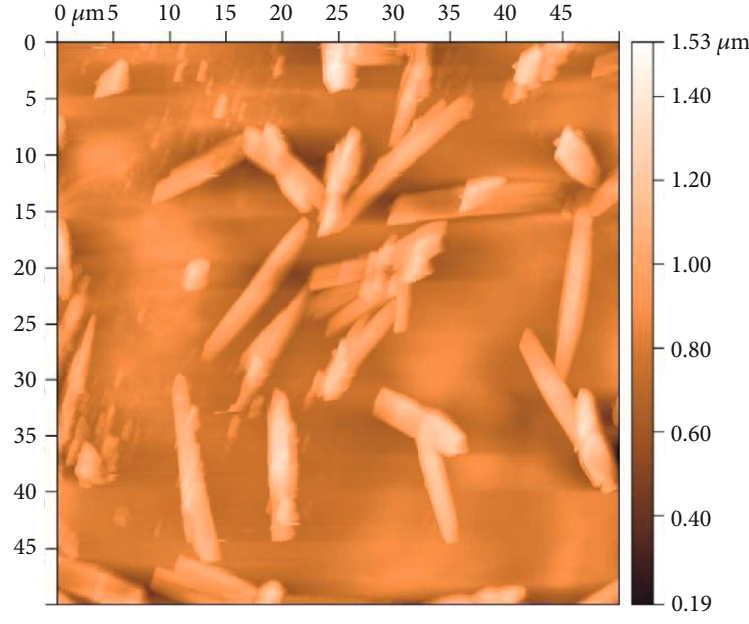

(a)

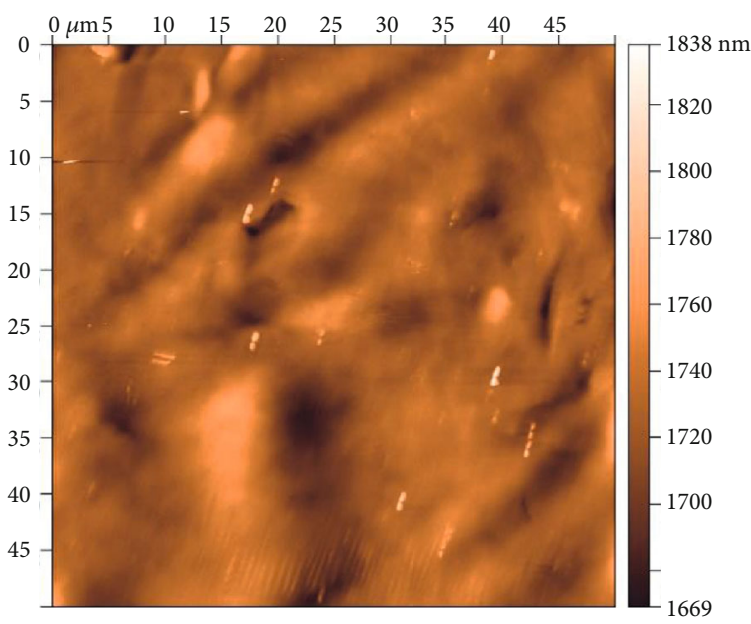

(c)

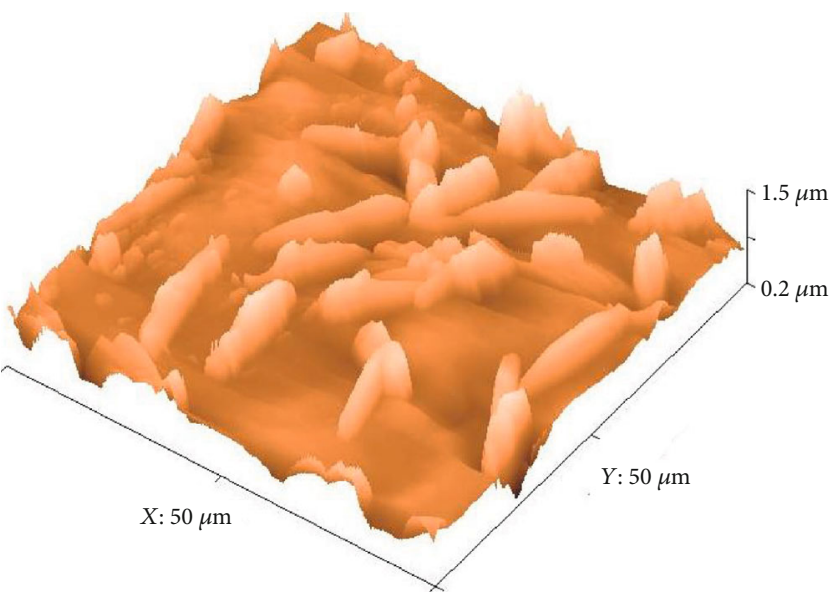

(b)

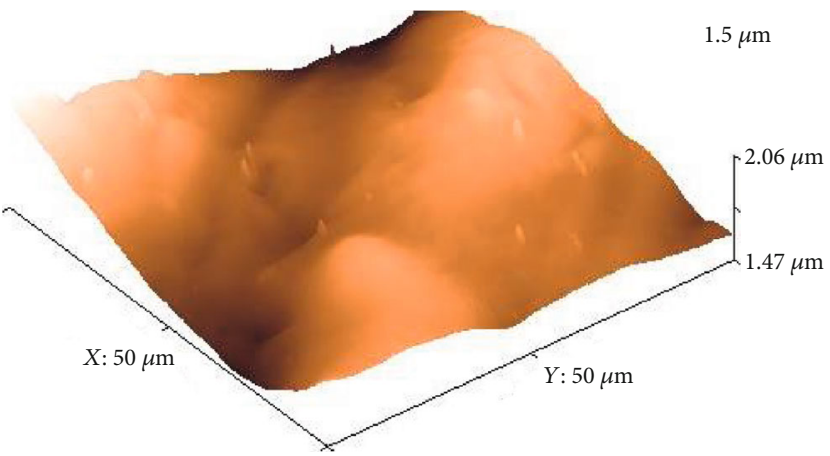

(d)

FIGURE 2: AFM micrograph of CNC film: (a) 2D-AFM micrograph of CNC film, (b) 3D-AFM micrograph of CNC film, (c) 2D-AFM micrograph of CNC with glycerol, and (d) 3D-AFM micrograph of the same sample.

acetylsalicylic acid as an active compound [15]. Acetylsalicylic acid formed crystals deposited on the HEC/PAAm composite surface [15]; the crystals also had resemblance to the crystals shown in Figures 3(a) and 3(b), although the crystals were formed by the active compound, as in the case of the ibuprofen on HPC/PAAm and not to the cellulosic compounds.

Figure 4 shows the profiles of the $\mathrm{CNC}$ film and of the $5 \mathrm{wt} \%$ film. Those profiles were obtained from the AFM micrographs, using the Gwyddion software. For the CNC film, Figure 4(a), an average height of $312 \mathrm{~nm}$ was found. The average diameter was measured to be $2.408 \mu \mathrm{m}$. The difference between the height measured by the profiles and the result obtained from the image may be due to the fact that the profile is measured in a line traced on the image, while the height measured directly on the image took into account as many protuberances as possible. For the CNC film obtained from a $5 \mathrm{wt} \%$ suspension, the profile of the sample is shown in Figure 4(b); this profile corresponds to the figures shown in Figures 3(a) and 3(b). The agglomerates are situated on the left part of the profile of the image. The height of the agglomerates is $88.71 \mathrm{~nm} \pm 17.05 \mathrm{~nm}$, while the measured diameter is $1268.12 \mathrm{~nm} \pm 195.58 \mathrm{~nm}$. The difference of the measurements of the diameter relative to those of Figure 3(a) is that the length measured from the profile was taken with a traced line that formed an angle close to $45^{\circ}$, so that it covered as many agglomerates as well as the substrate shown in Figure 3(a). The diameter measured from the profile in Figure 4 is close to the measurement of the hypotenuse of a right-angled triangle, $c^{2}=a^{2}+b^{2}$, using the values of length and diameter measured in Figure 3(a). The thickness of the layer was measured by finding a plateau in the profile; this plateau was the lowest part of the agglomerates. The average measured thickness of the layer was $156.11 \mathrm{~nm} \pm 4.85 \mathrm{~nm}$, and the average height of the layer was $244.82 \mathrm{~nm}$.

The electric behaviour of cellulose is summarized in Table 2. The CNC sample has a dielectric strength similar to the dielectric industrial paper. The CNC sample had no additives; it is interesting to note that its dielectric strength matches the value of industrial dielectric paper. It was found that the humidity of the $\mathrm{CNC}$ films lowered dielectric 


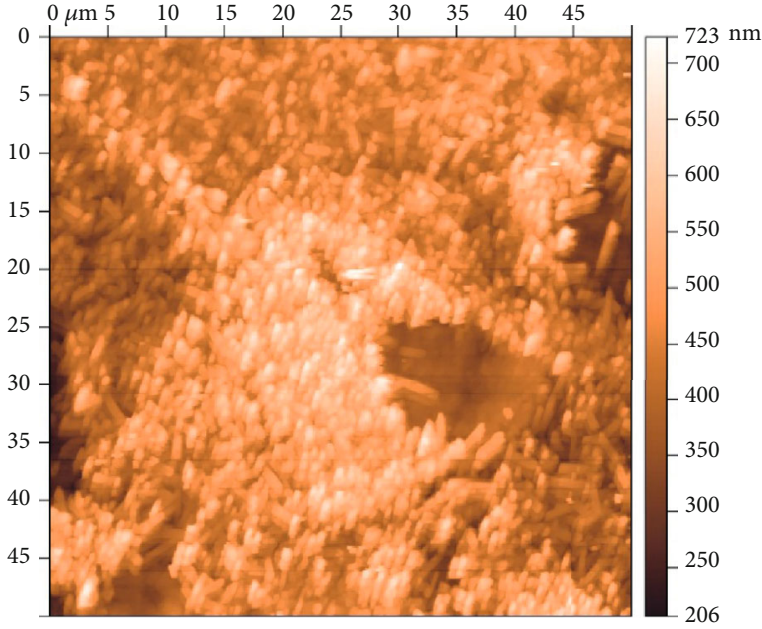

(a)

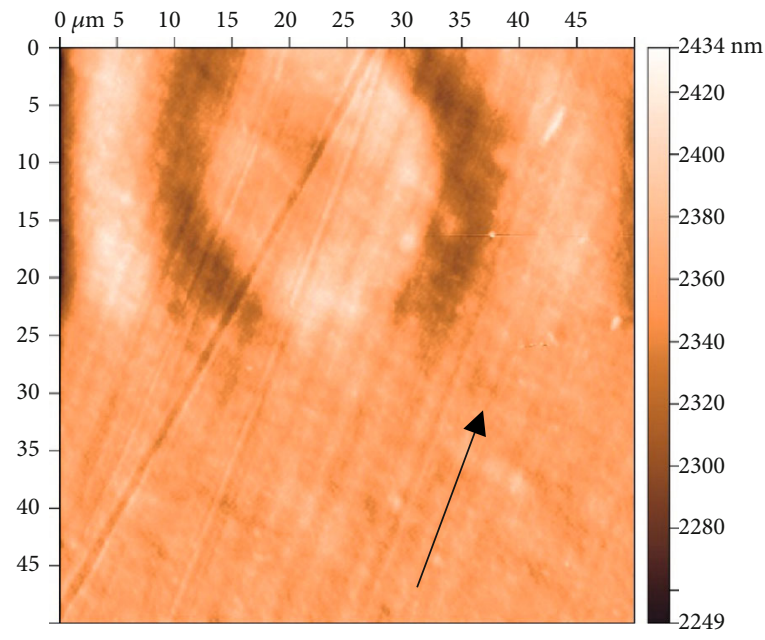

(c)

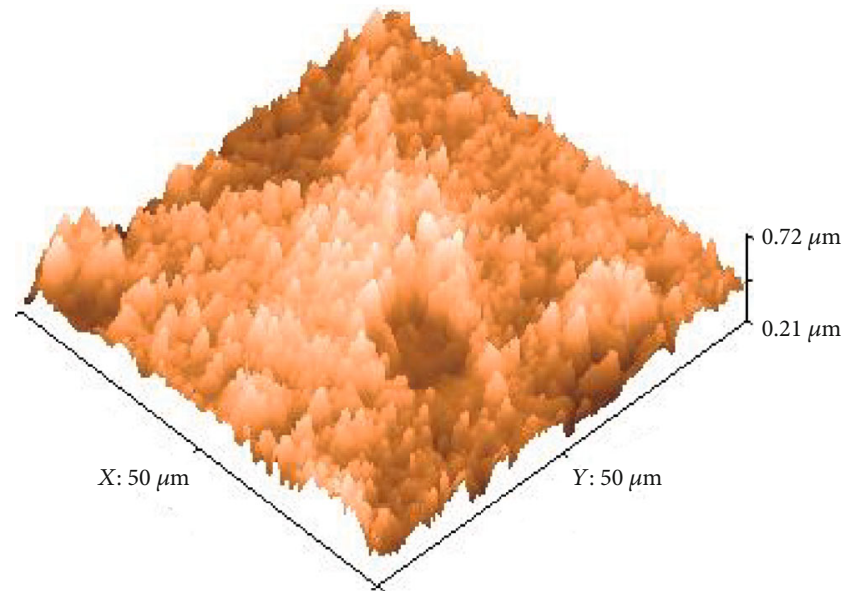

(b)

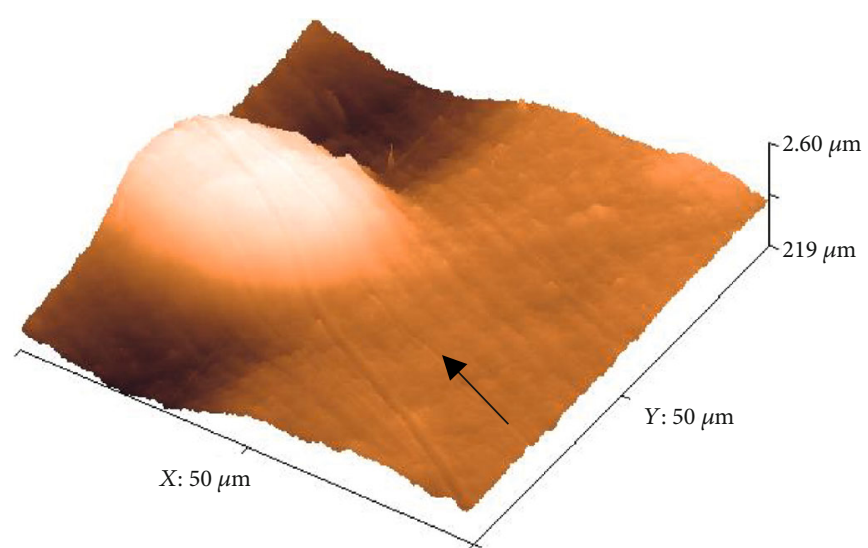

(d)

FIgURE 3: AFM micrograph of CNC film formed with a $5 \mathrm{wt} \%$ CNC sample. (a, c) 2D-AFM micrographs of the sample and (b, d) 3D-AFM micrographs of the same sample.

strength; to avoid humidity on the samples, the CNC suspensions were dried in an oven for 5 days and then placed on a desiccator; if these steps were not done, humidity entered the CNC films. Moisture content affects the insulating behaviour of materials [16]. The dielectric strength of the paper diminished with the addition of glycerol. The role of glycerol was to increase the flexibility of CNC films, as they are fragile in the pure state. We observed that the CNC film with glycerol was more flexible and less fragile, but the dielectric strength diminished significatively. This was attributed to the presence of glycerol, which had a plasticizing effect on the CNC films; plasticizer compounds increase the mobility of polymer chains. It is considered that plasticizers embed themselves between the polymer chains, increasing the free volume. The presence of the plasticizing between the CNC chains makes the path of electricity easier. In our finds, the glycerol interfered with the dielectric properties of $\mathrm{CNC}$, consistently with theoretical considerations.

The CNC films fabricated with CNC solutions with a concentration of $5 \mathrm{wt} \%$ also had a low dielectric strength.
CNC is known to arrange in a helicoidal pattern at high concentrations, forming a chiral nematic phase. When an electrical field is present, the nanocrystals tend to align in a given direction, without forming the helicoidal pattern; this is due to the Fréedericksz transition. The attempt to form a film with a highly concentrated $\mathrm{CNC}$ solution was aimed at avoiding the chiral nematic pattern and uniformly orienting the crystals, thus reducing the probability of an electrical current of passing through the polymer chains. However, in our findings, the dielectric strength is lower than that of the pure CNC film. We think that the result may be explained by the fact that the crystals orientated with the electric field, with a preferred direction of alignment. However, the chiral nematic phase may make the pass of the electrical current in the film difficult, as each layer has an orientation that is rotated with respect to the adjacent layers. The rotation of the layers makes the current rebound in directions different to the preceding layer, making the electrical current to pass for a longer length than with a uniformly oriented crystal, where the angle of deviation would be lower than the 


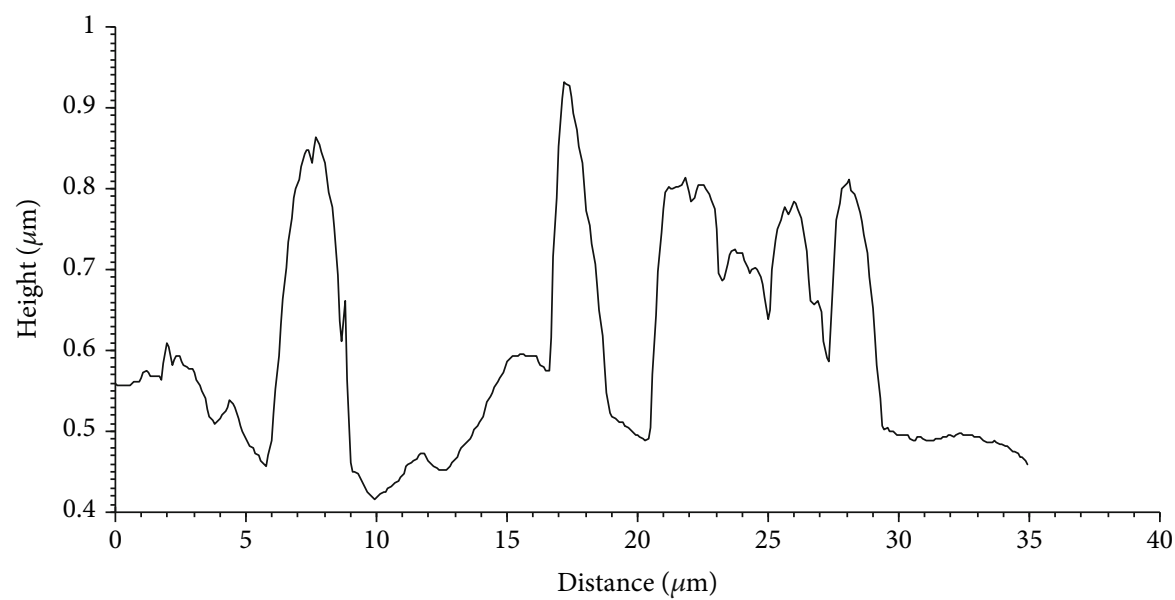

(a)

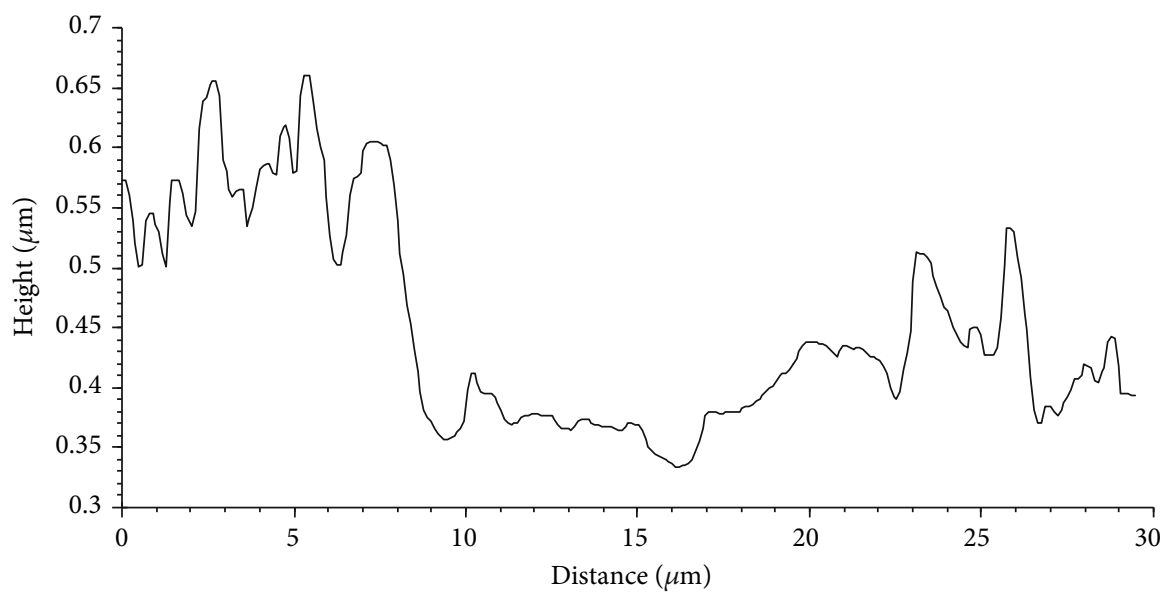

(b)

Figure 4: Profiles of the CNC samples. (a) CNC film and (b) CNC film formed with a $5 \mathrm{wt} \%$ CNC suspension.

TABLE 2: Dielectric properties of cellulosic samples at $25^{\circ} \mathrm{C}$.

\begin{tabular}{lcc}
\hline Sample & $\begin{array}{c}\text { Dielectric strength } \\
(\mathrm{kV} / \mathrm{cm})\end{array}$ & $\begin{array}{c}\text { Thickness } \\
(\mu \mathrm{m})\end{array}$ \\
\hline Commercial dielectric paper & 187 & 80 \\
CNC sample & 189 & 20 \\
CNC sample not in desiccator & 68 & 55 \\
CNC glycerol & 38 & 110 \\
CNC 5 wt\%, electric field & 18 & 130 \\
\hline
\end{tabular}

deviation angle caused by a chiral nematic phase. This is illustrated in Figure 5; Figure 5(a) shows the pass of an electrical current in a cellulose nanocrystal compound, having a chiral nematic arrangement. Cellulose nanocrystals are known to retain the chiral nematic arrangement upon drying $[17,18]$. The cholesteric phase has layers that are twisted in respect of the adjacent layers, thus making the material with different directions; this effects force the electrical current to find zones that offer less resistance to the pass of the current. On the other hand, Figure 5(b) shows a crystal with long-range orientation and no twist. As the crystals are oriented in the same direction, there is less deviation and thus less resistance to the pass of the electrical current.

The results of the dielectric strength may be of interest to the electrical industry, as CNCs can form compounds with the same dielectric strength of industrial dielectric paper, but with improved mechanical and chemical properties, as the higher crystallinity of CNCs improves those properties. Flexible compounds made from $\mathrm{CNC}$, which retain the dielectric properties, would be a new material of industrial interest. CNCs are a good material for improving and substituting current dielectric paper in the parts where they are used on the products of the energy sector.

The crystalline arrangement of the dried films was studied using polarized optical microscopy; Figure 6 shows an image of the film observed under crosspolarizers. The sample shows iridescence, typical of cellulose nanocrystals [19]. The iridescence of the sample is seen as different colours in the image; they are caused by the cellulose nanocrystals of the film, which interact with the light of the microscope, although the sample does not show any pattern characteristic of a chiral nematic phase. The concentration of the starting solution is below the value of the concentration where the chiral nematic phase forms. However, the features observed in the image resemble 


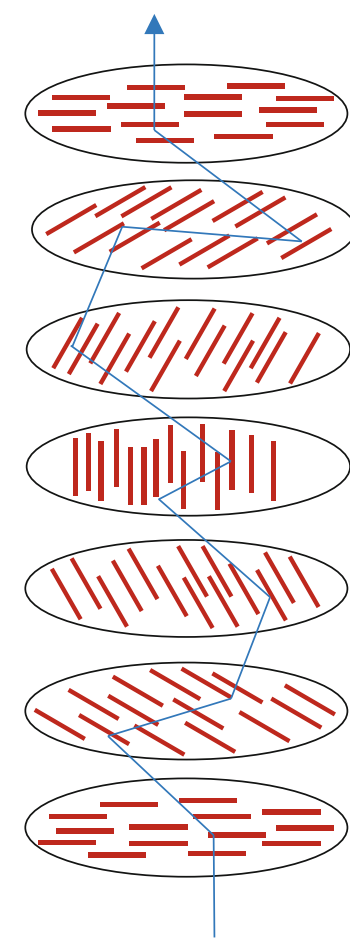

(a)

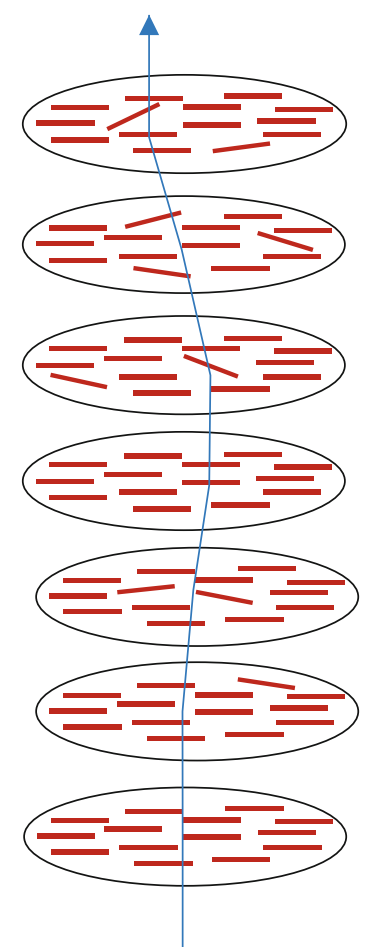

(b)
FIGURE 5: Scheme of the pass of an electrical current through (a) a chiral nematic crystal and (b) a crystal having the same orientation on its layers. For the chiral nematic crystal, an arrangement of a pitch $\mathrm{p} / 2$ is shown.

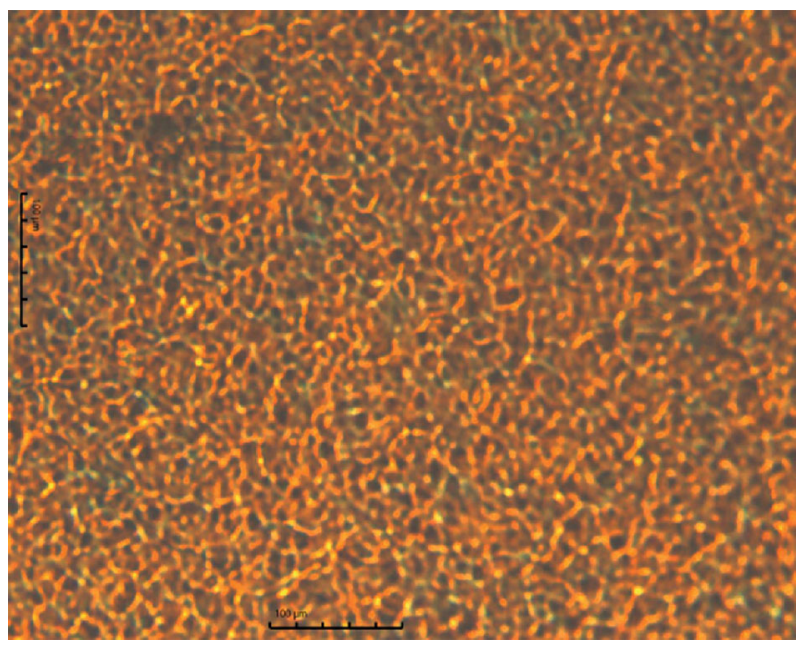

Figure 6: Polarized optical microscope of a dried film, seen under crosspolarizers. Scale bar is $100 \mu \mathrm{m}$.

the texture of oily streaks, a texture found on chiral nematic phases of cellulose derivatives; this suggests that during drying, there is an ordering of the cellulose nanocrystals.

Figure 7 shows an image of a CNC film observed with different angles of light incidence. The film shows iridescence that can be seen in Figures 7(a), 7(b), and 7(d), with different colours being reflected on the surface of the film, which is noted in Figures 7(c) and 7(d). This suggests that upon drying, the cellulose nanocrystals had the time to self-organize and have an ordered arrangement, as the iridescence indicates the result of constructive light interference caused by the ordered cellulose nanocrystals in the film [20]. There are in the literature other reports of iridescence of CNC films dried on polystyrene Petri dishes [13, 21]. The observed iridescence and different colours on the dried film confirm that the cellulose nanocrystals of the film have an ordered arrangement, or else the film would be colourless, would have no interference, and would not display different colours when the angle of light is varied. The order of the cellulose nanocrystals in the dried film has an effect on the dielectric strength of the CNC film.

Figure 8 shows the thermal degradation behaviour of the cellulosic samples. The CNC film sample losses $6 \%$ of humidity at $100^{\circ} \mathrm{C}$; this humidity is due to nonevaporated water that was trapped inside the polymer layers. The sample remains stable on heating and starts to degrade at around $250^{\circ} \mathrm{C}$; before that temperature, there is no significant degradation of the cellulose nanocrystals. The onset temperature of the CNC film is similar to samples observed in literature [22]. The observed degradation behaviour is typical of cellulose [23] and of cellulose derivatives [24], with a sharp decrease starting at the temperature of degradation and then reaching a plateau. At $400^{\circ} \mathrm{C}$, the residue is at around $11 \%$, this residue is mainly due to the presence of the decomposition product of cellulose, levoglucosan [25]. The levoglucosan is then decomposed during heating; then, it starts to slowly decompose in low-molecularweight hydrocarbons, until another plateau is reached at the end of the analysis at $750^{\circ} \mathrm{C}$; the final residue is $3.27 \%$. The TGA indicates that there is some humidity on the film. Humidity has the effect of lowering the dielectric strength [11], as the water molecules within the film open a path for the electrical current. The dielectric strength may be improved if the humidity content of the film was decreased. The TGA of the dielectric paper is shown in the same figure. The sample starts to degrade at $280^{\circ} \mathrm{C}$; then, it follows a degradation course typical of cellulosic samples [23] and reaches a plateau at $380^{\circ} \mathrm{C}$. The final residue is around $22 \mathrm{wt} \%$. The weight loss of the dielectric paper is faster than the weight loss of the CNC film, as CNC samples show a more complex thermal behaviour due to the sulfate groups on the surface of the $\mathrm{CNC}$ crystals. The dielectric paper degrades at a higher temperature; CNC degrades at lower temperature, as the sulfate groups of $\mathrm{CNC}$ are labile, but the dielectric paper has additives to improve its performance in power transformers and to withstand the temperatures of the power transformer; this is the reason why the residue of the dielectric paper is higher than that of the CNC sample.

Figure 9 shows the SEM micrograph of the pure CNC film and under different processing. Figure 9(a) shows the SEM micrograph of the pure CNC film. The film has a surface covered by rods formed by CNC particles, which look like the rods shown by AFM in Figure 2. The SEM micrograph confirms the presence of the micrometric rods on the film surface. The dimensions of the rods were measured using the Gwyddion software, and the length was $5.68 \mu \mathrm{m} \pm 0.34 \mu \mathrm{m}$, 


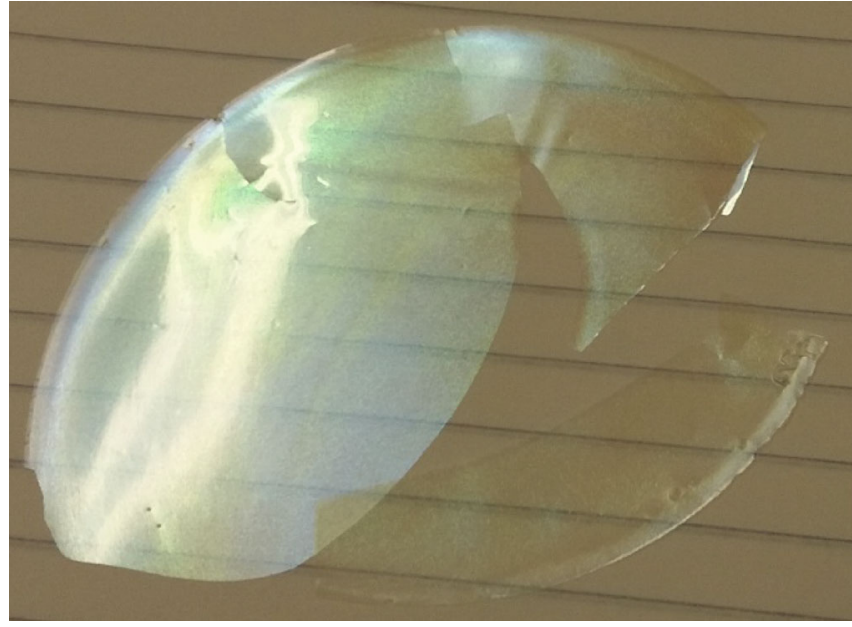

(a)

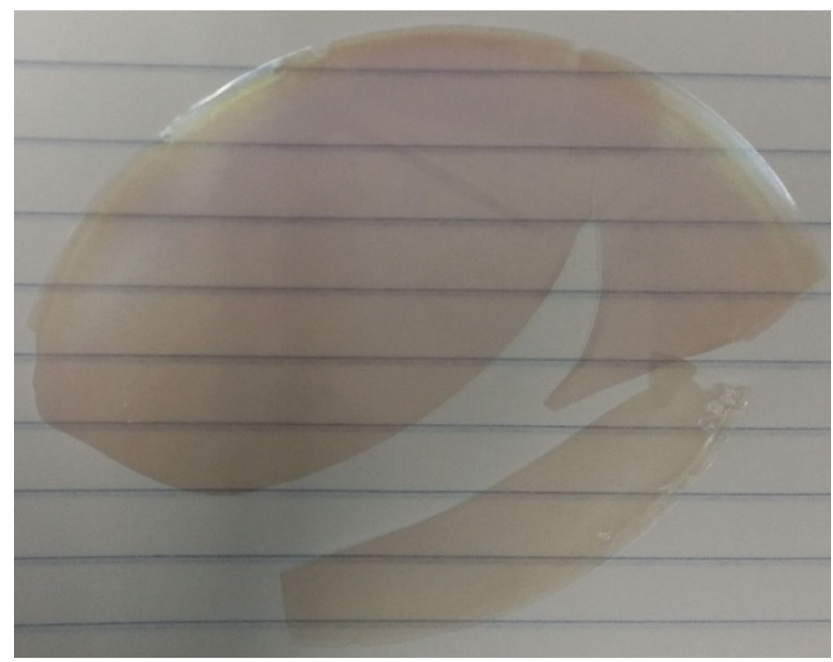

(c)

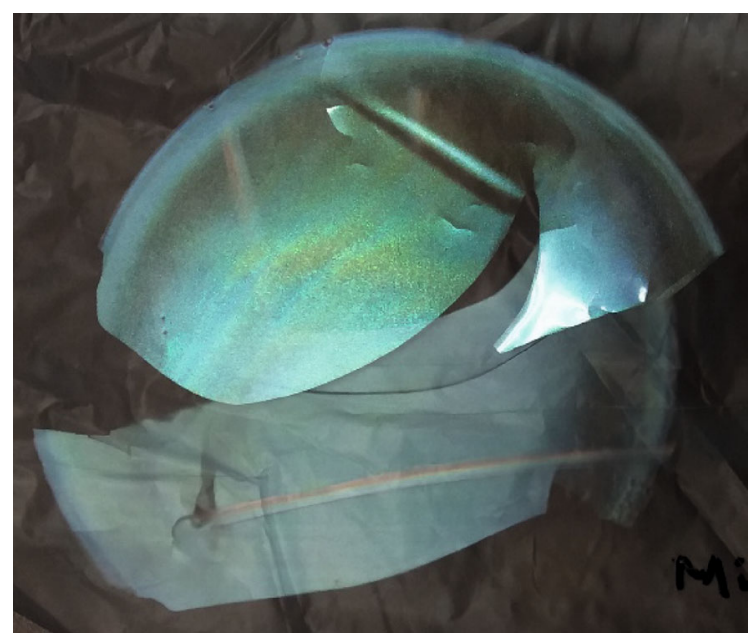

(b)

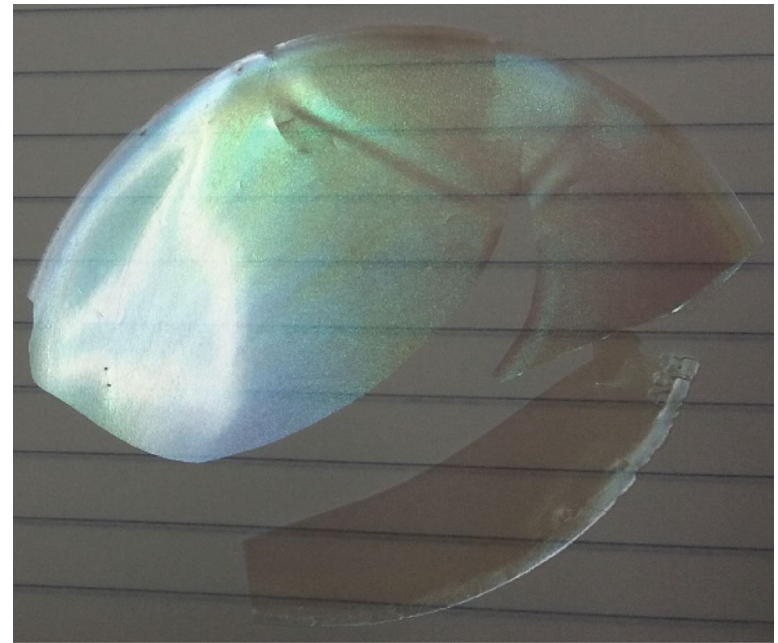

(d)

Figure 7: CNC film. The film has iridescence (a, b, and d), and the colour of the film changes when the angle of the light is changed (c, d). The lines on $(c, d)$ are a reference to see the angle of the picture.

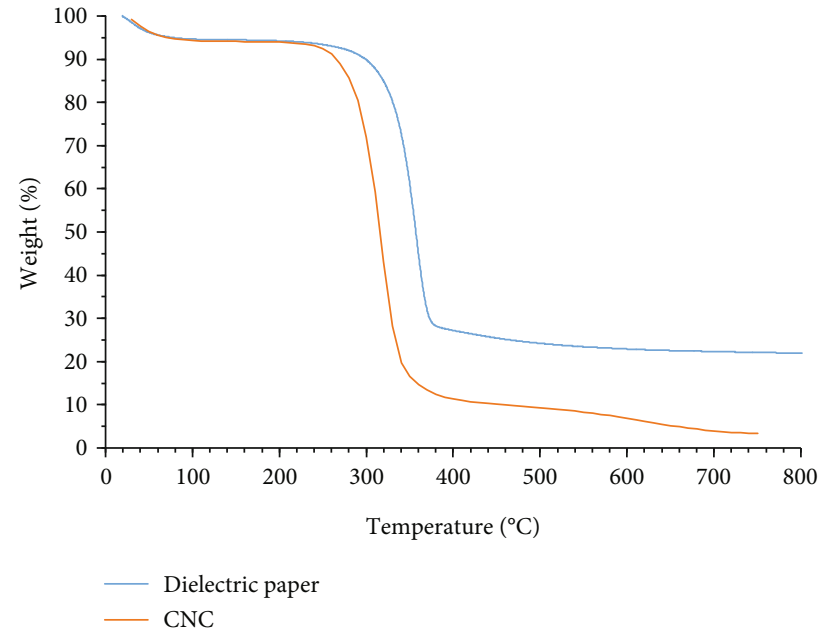

Figure 8: TGA curve of cellulosic samples. while the diameter was $671 \mathrm{~nm} \pm 19.94 \mathrm{~nm}$. The dimensions measured by the SEM are lower than those obtained by the AFM. This can be explained as the dimensions obtained by SEM are usually lower due to some effects like the tip convolution and the interaction between the AFM tip, film surface, and particle that produces a broadening of the imaged particles [26]. The difference of the sizes might be due to different areas of the sample being imaged.

Figure 9(b) shows the SEM micrograph of the CNC film with glycerol. The surface of the film is mostly smooth, without the rods observed on the CNC film. Some features are also observed on the surface, due to the flexibility of the film. The features observed are small lines and a groove on the film.

Figure 9(c) shows the SEM micrograph of the $5 \mathrm{wt} \% \mathrm{CNC}$ film. The film looks like the AFM image in Figure 3. Parallel lines are also observed on the surface of the film, like those observed in Figure 3(c). Some agglomerates can be seen on the surface, at the lower right corner of the micrograph, like those observed in Figure 3(a). The SEM image confirms the 


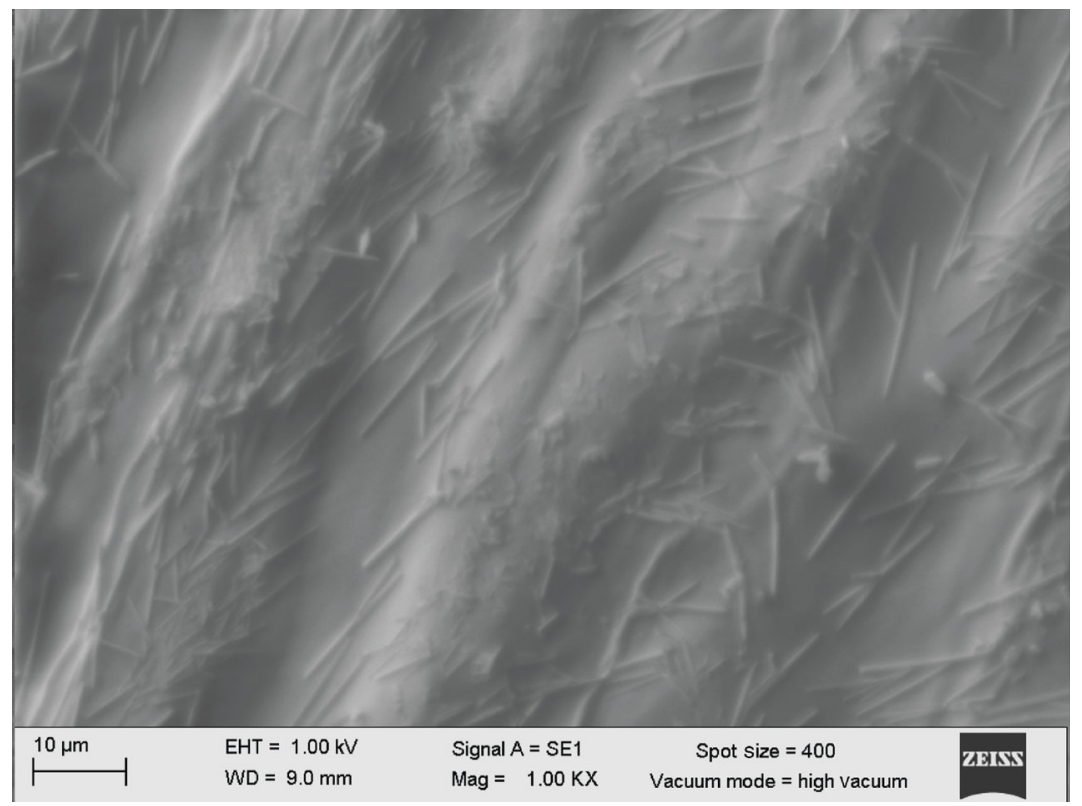

(a)

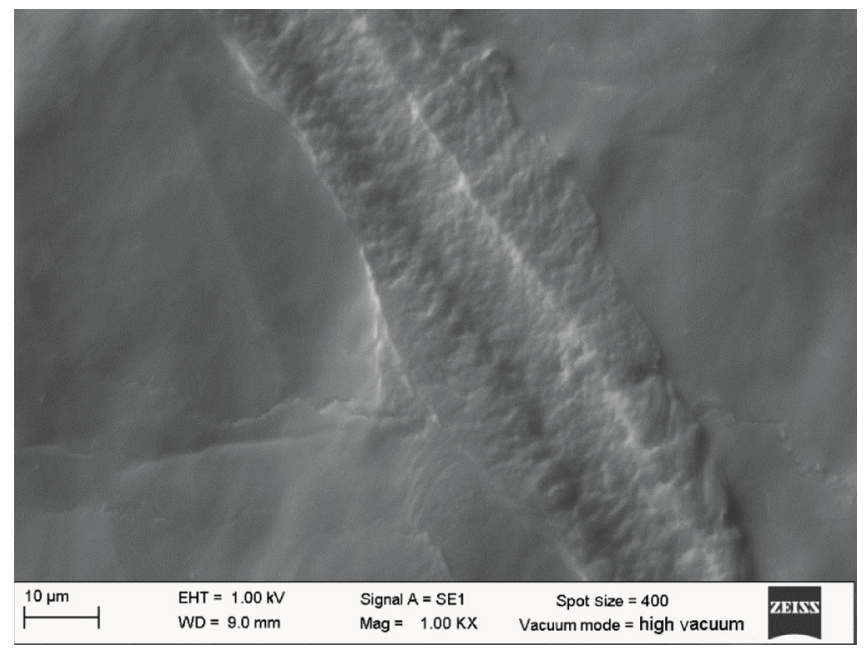

(b)

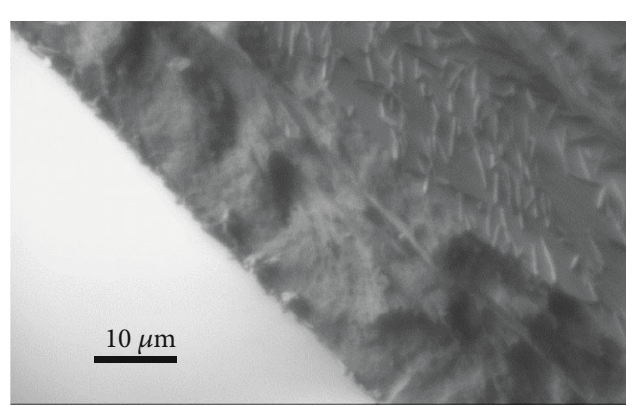

(d)

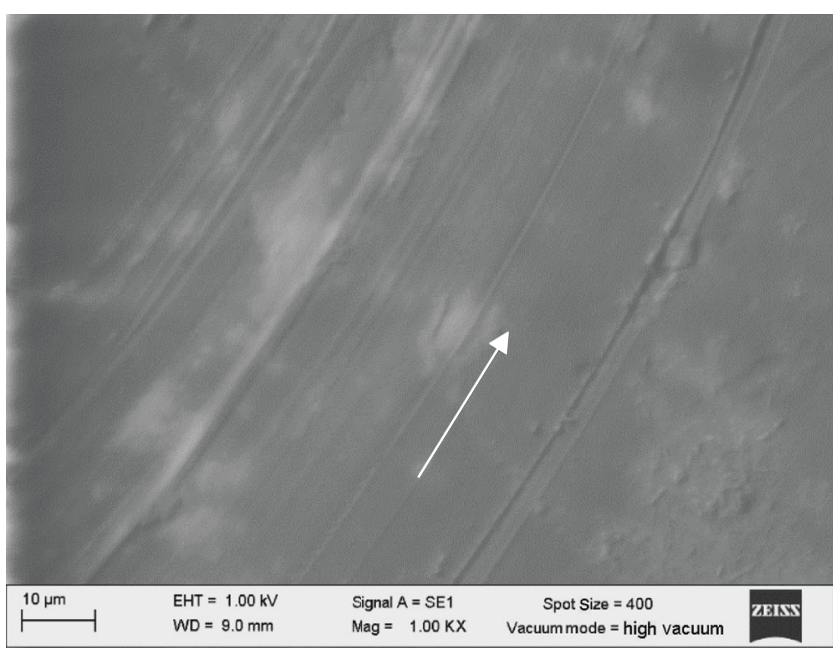

(c)

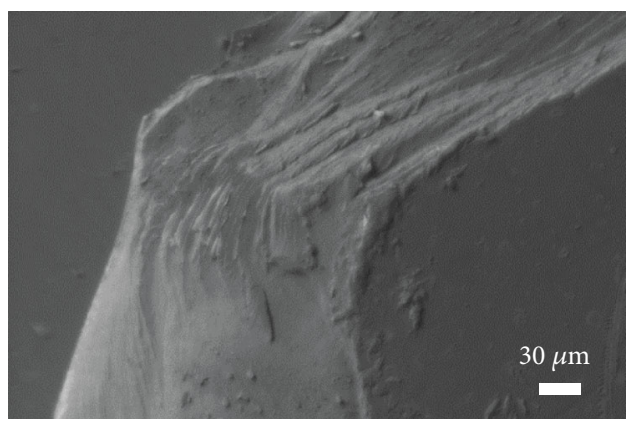

(e)

Figure 9: SEM micrograph of CNC films: (a) pure CNC film, (b) CNC film with glycerol, (c) 5 wt\% CNC film, (d) lateral view of pure CNC film, and (e) lateral view of $5 \mathrm{wt} \% \mathrm{CNC}$ film.

presence of the features observed in Figure 3. The orientation of lines in Figure 9(c) is marked with an arrow.

Figure 9(d) shows a micrograph of the edges of the CNC film and Figure $9(\mathrm{e})$ the edges of the CNC $5 \mathrm{wt} \%$. Those figures correspond, respectively, to the samples of Figures 9(a) and 9(c). The films were cut and then put over carbon tape. The thickness of the films can be obtained from the figure. For the $5 \mathrm{wt} \%$ sample, the thickness of the film is $175 \mu \mathrm{m}$; for 


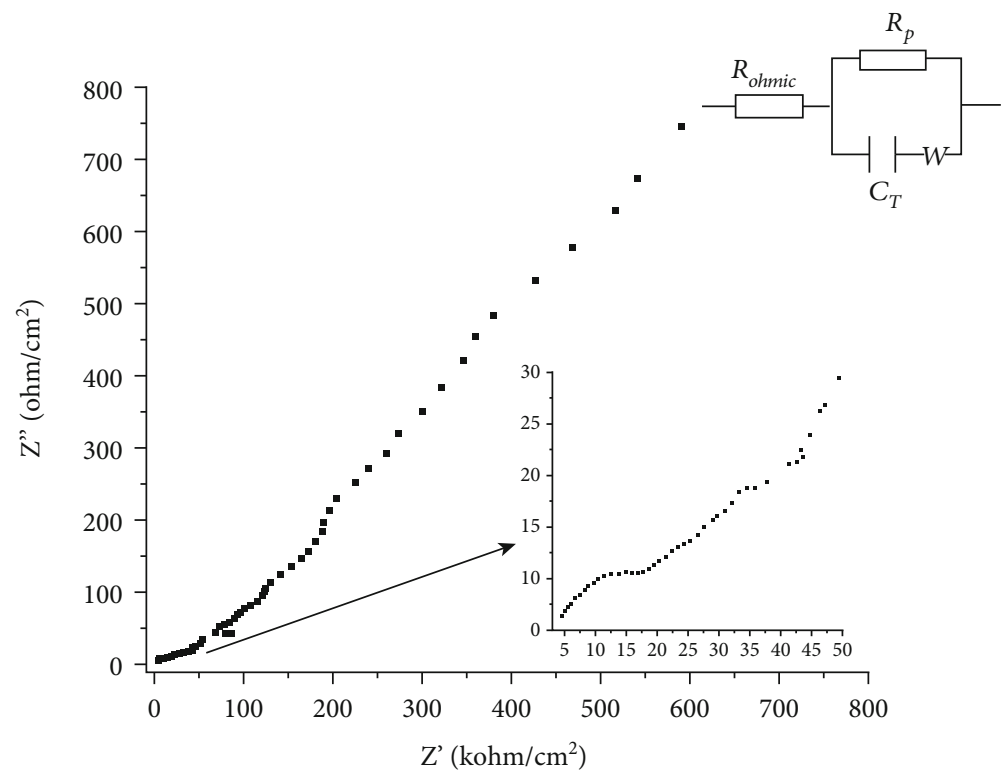

Figure 10: Nyquist diagrams for the device with CNC.

TABle 3: Fitting parameters for EIS spectrum of the device.

\begin{tabular}{lc}
\hline Element & Value \\
\hline$R_{\text {ohmic }}\left(\mathrm{kohms} / \mathrm{cm}^{2}\right)$ & 2.79 \\
$\mathrm{CPE}_{\mathrm{T}} Y_{0}\left(\mathrm{kohms}^{-1} \mathrm{~s}^{n}\right)$ & $3.07 \times 10^{-8}$ \\
$\mathrm{CPE}_{\mathrm{T}} n$ & 0.6 \\
$R_{\mathrm{p}}\left(\mathrm{kohms} / \mathrm{cm}^{2}\right)$ & 37.47 \\
$W R_{\mathrm{w}}\left(\mathrm{kohms} / \mathrm{cm}^{2}\right)$ & 135.26 \\
$s$ & 0.02 \\
$\alpha$ & 0.25 \\
\hline
\end{tabular}

the CNC sample, the thickness is $29 \mu \mathrm{m}$. The reason for the difference of the thickness of the films is the concentration of the samples used for evaporation. Factors such as concentration, volume, and sample preparation are reported in literature to influence and determine the thickness of CNC films [27]. The profile of sample CNC $5 \mathrm{wt} \%$ is interesting, because the edge shows parallel lines, with an average separation of $1.97 \mu \mathrm{m}$. The parallel lines are formed by the ordering of the layers of CNC particles that occurs in the axis perpendicular to the surface of the film. CNC samples show the ordering of the particles on edges of films [28]; the ordering of the chiral nematic phase of CNCs can be observed at higher magnifications at the edges of the films [28]. In the case of our concentrated films, the CNCs are not arranged in a chiral nematic phase, but the aligning of the sample is in a given direction, as a magnetic field was applied to the sample. The lines observed on the upper side of Figure 9(e) are parallel to the surface of the film, suggesting that the orientation axis is perpendicular to the surface [28].

In order to corroborate that a CNC film has a good dielectric capacity, a capacitor-type plastic film was constructed. The plastic film capacitors have plastic films and metal sheets interspersed and have characteristics that make them suitable for various applications: filtering, decoupling, bypassing, EMI suppression, pulse coupling, blocking, and smoothing [29]. Plastic film capacitors are very stable over a wide temperature range with good reliability and long life expectancy [30].

Figure 10 shows the Nyquist diagrams for the device. At high-frequency values, a partially capacitive-like depressed semicircle was observed (inserted figure). The semicircle is the result of the combination of a resistance; in this case, it is a polarization resistance, which is the sum of the charge transfer resistance in the metal/CNC interface in parallel to a total capacitance, which is the sum of the capacitances in the said interface. Also, this semicircle has been associated with a surface of the electrodes with defects such as pores [31]. At low frequencies, another behaviour was observed; a straight line inclined slightly to the imaginary axis. This behaviour is indicative of the good ability of the device to store charge on the electrode surface [32].

An equivalent circuit (Figure 10, inset) was used to simulate the impedance data. In the circuit, $R_{\text {ohmic }}$ is the ohmic resistance related to the ohmic losses in the device which are mainly due to the contact resistances between interfaces [33]. This resistance is the intersection of the partial semicircle with the real axis $\left(Z^{\prime}\right)$ at high frequencies; $C_{\mathrm{T}}$ is the total capacitance, $R_{\mathrm{p}}$ is the polarization resistance (intersection of partial semicircle at middle frequencies), and finally, $W$ is the Warburg impedance, which was used to simulate the behaviour observed at low frequencies. Instead of a capacitive element $(C)$, a constant phase element $(\mathrm{CPE})$ is used to have a better fit of the data and because the surface of the electrodes is not homogeneous [34] (roughness, dislocations, impurities, among others) [35]. The simulated parameter values are presented in Table 3.

The high values of the polarization resistance indicate that the charges accumulated on the surface of the electrodes have great difficulty in being inserted or removed from the 

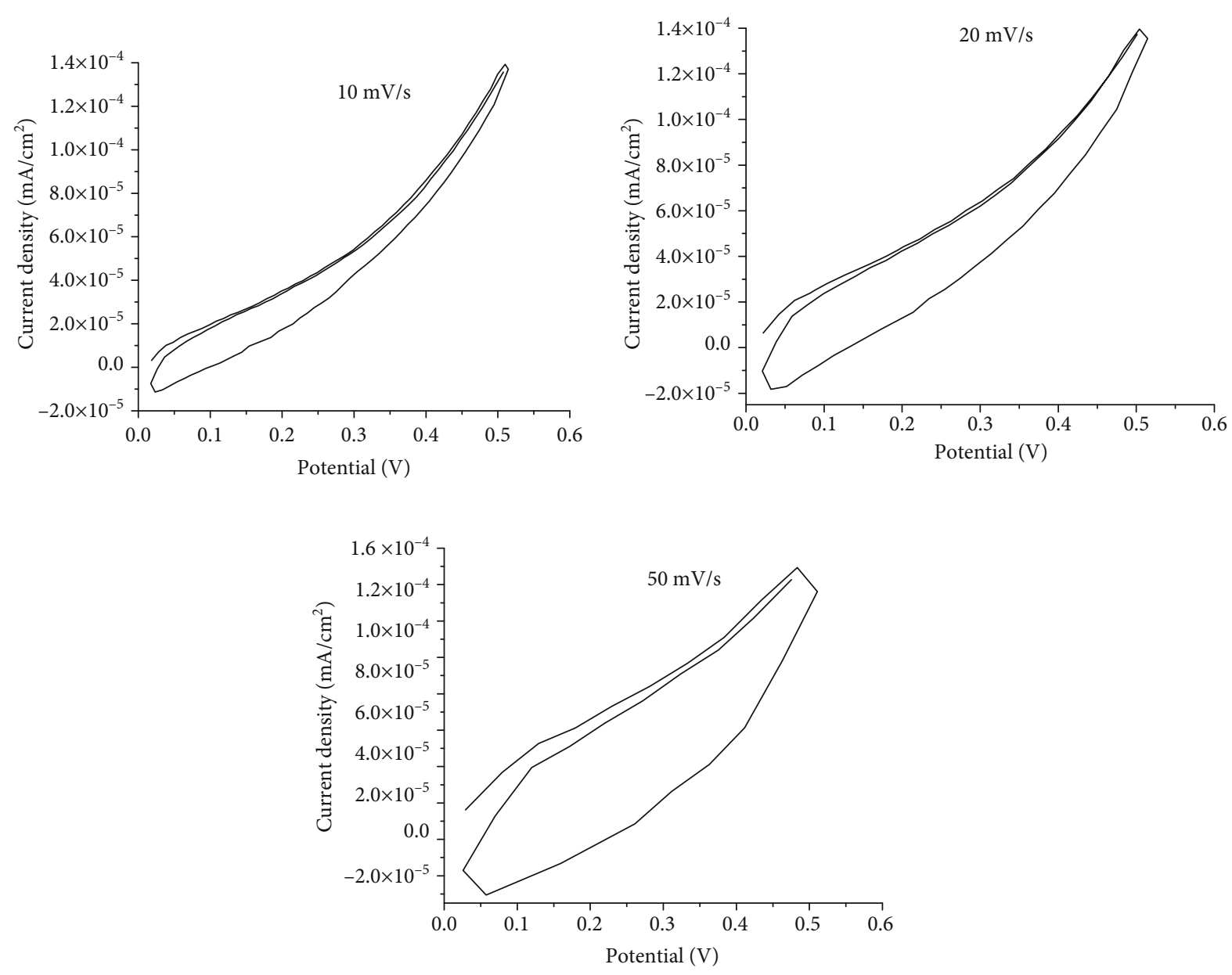

FIgURE 11: CV curves for the CNC device at different scan rates.

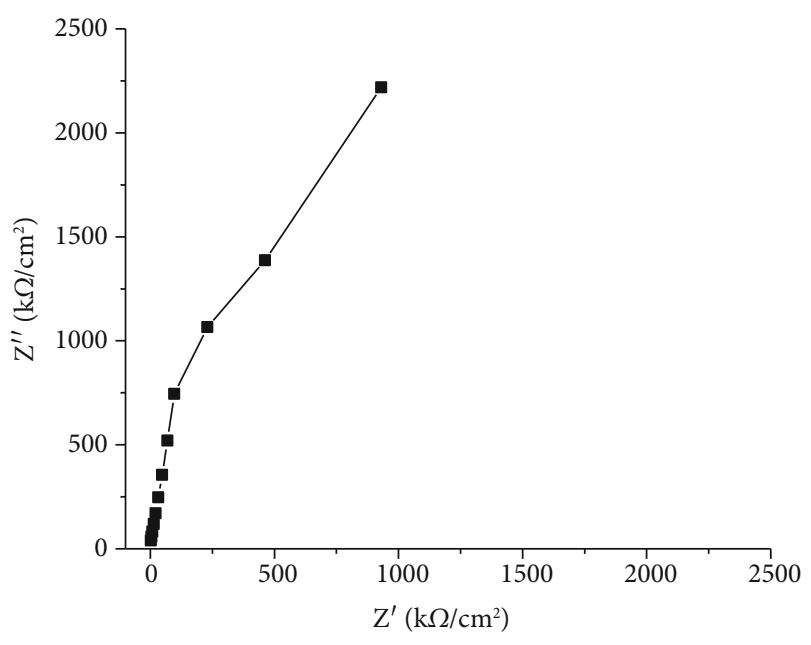

Figure 12: Nyquist diagrams for the device with dielectric paper.

dielectric barrier [36]. This verifies that the $\mathrm{CNC}$ has a good capacity as a dielectric material and can be used in this type of application.

Figure 11 shows the cyclic voltammetry $(\mathrm{CV})$ curves for the device at different scan rates: $10 \mathrm{mV} / \mathrm{s}, 20 \mathrm{mV} / \mathrm{s}$, and
$50 \mathrm{mV} / \mathrm{s}$. All the CV curves exhibit quasirectangular shapes, indicating that the electrode material and $\mathrm{CNC}$ film have potential application in capacitors due to their performances $[37,38]$. The best performance according to the shape of the curve was obtained with the scan rate of $50 \mathrm{mV} / \mathrm{s}$. At lower scan rates $(10 \mathrm{mV} / \mathrm{s}$ and $20 \mathrm{mV} / \mathrm{s})$, the shape of the curve moves slightly away from the rectangular shape. This behaviour may be due to a faradaic capacitance [39] and to the electron transfer process [40], which in this case is limited at $10 \mathrm{mV} / \mathrm{s}$ and $20 \mathrm{mV} / \mathrm{s}$.

In the case of dielectric paper, a behaviour similar to that of CNC was observed, a partially capacitive-like semicircle at high frequencies and a quasivertical line at medium-low frequencies (Figure 12). However, the polarization resistance is higher $\left(2278 \mathrm{kohms} / \mathrm{cm}^{2}\right)$ compared to the value obtained $\left(37.47 \mathrm{kohms} / \mathrm{cm}^{2}\right)$ with the CNC device. This behaviour may indicate that the dielectric paper has fewer surface defects compared to the CNC device and its function as a barrier is more efficient. However, the polarization resistance values and the ability to accumulate charges on the electrode surface of both materials are preferred for different applications. The equivalent circuit of Figure 12 was used to simulate the impedance data. The simulated parameter values are presented in Table 4. 
TABLE 4: Fitting parameters for EIS spectrum of the devices.

\begin{tabular}{lc}
\hline Element & Value \\
\hline$R_{\text {ohmic }}\left(\mathrm{kohms} / \mathrm{cm}^{2}\right)$ & 3.07 \\
$\mathrm{CPE}_{\mathrm{T}} Y_{0}\left(\mathrm{kohms}^{-1} \mathrm{~s}^{n}\right)$ & $1.79 \times 10^{-8}$ \\
$\mathrm{CPE}_{\mathrm{T}} n$ & 0.8 \\
$R_{\mathrm{p}}\left(\mathrm{kohms} / \mathrm{cm}^{2}\right)$ & 2278 \\
$W R_{\mathrm{w}}\left(\mathrm{kohms} / \mathrm{cm}^{2}\right)$ & 273.96 \\
$s$ & 0.03 \\
$\alpha$ & 0.66 \\
\hline
\end{tabular}

Figure 13 shows the CV curves for the device with dielectric paper at $10 \mathrm{mV} / \mathrm{s}, 20 \mathrm{mV} / \mathrm{s}$, and $50 \mathrm{mV} / \mathrm{s}$. All the $\mathrm{CV}$ curves exhibit a quasirectangular shapes very similar to that observed with $\mathrm{CNC}$ at $50 \mathrm{mV} / \mathrm{s}$. In this case, the faradaic capacitance and the electron transfer process are not limited at different scan rates.

\section{Conclusions}

Development of new materials in the electric industry opens opportunities to improve both equipment and machines into the power system. CNC films are a good option to be used as dielectric materials in the energy sector, given its good mechanical and thermal properties. The CNC films showed a dielectric strength that was equal to the value of industrial insulating paper, suggesting that CNCs may be a material used for fabrication of dielectric parts. As CNC films could reduce its thickness up to four times, opportunities are opened specially to gain cross-sectional area in slots of small rotating machines. The more available area in slots, the more efficiency of the machine. During fabrication of the films, there should be care on the manipulation of the films, and on the surrounding atmosphere, as moisture lowers the dielectric strength. Glycerol reduced the fragility of the films, but it lowered the value of dielectric strength. The effect of an electrical field on concentrated films lowered the value of the dielectric strength of the CNC films. The dielectric strength may be improved if CNCs are used for fabricating sheets of CNC. The films had a content of moisture on them; if the value of humidity was lower, the dielectric strength of the material may be higher. The films showed iridescence; this suggests that the cellulose nanocrystals were ordered in the dry state. This phenomenon may increase the dielectric strength of the CNC films, as it makes the electrical current to find clearer zones for it to pass. The film synthesized from a $5 \mathrm{wt} \%$ suspension and exposed to an electrical field had an oriented morphology; however, its dielectric strength was lower than that of the CNC film. It is theorized that the layered orientation of the CNC films will make the pass of the electrical current difficult. SEM characterization confirmed the results of AFM, with some rods observed on the CNC films; the thickness of the films could be measured using this technique. The arrangement of the layers of CNC particles of the $5 \mathrm{wt} \%$ film could be observed by SEM, suggesting that the axis is arranged per-
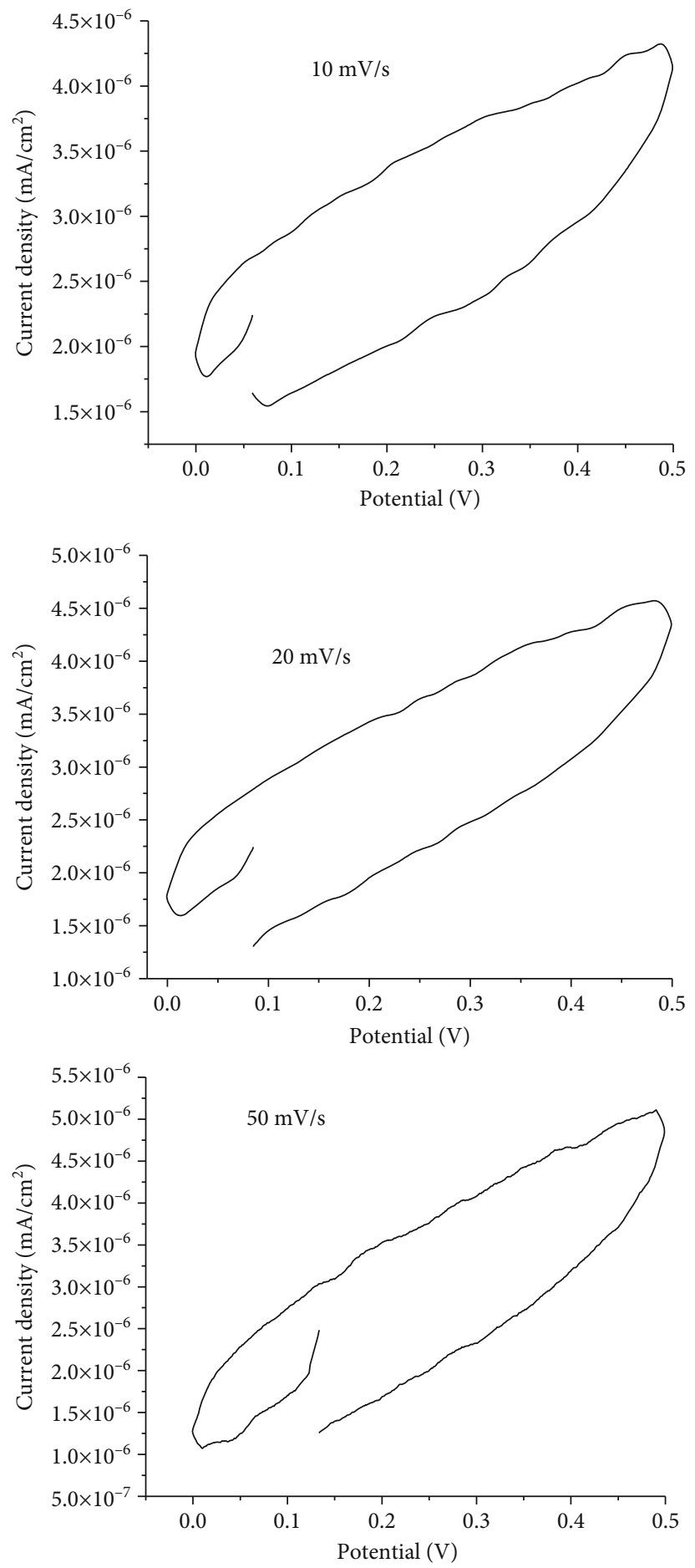

FIgURE 13: CV curves for the device with dielectric paper at different scan rates.

pendicularly to the surface of the film. The results of the capacitor device suggest that $\mathrm{CNC}$ films have potential for capacitor fabrication and that the films are a good dielectric material. The best performance of the devices was obtained using a scan rate of $50 \mathrm{mV} / \mathrm{s}$, with a deviation of a rectangular shape at lower scan rates, a behaviour caused by faradaic capacitance and by electron transfer processes. 


\section{Data Availability}

The data used to support the findings of this study are included within the article.

\section{Conflicts of Interest}

The authors declare that there are no conflicts of interest.

\section{Acknowledgments}

This study was supported by CONACyT. We thank INEEL for allowing us to use their facilities.

\section{References}

[1] D. Klemm, F. Kramer, S. Moritz et al., "Nanocelluloses: a new family of nature-based materials," Angewandte Chemie, International Edition, vol. 50, no. 24, pp. 5438-5466, 2011.

[2] Y. Habibi, L. A. Lucia, and O. J. Rojas, "Cellulose nanocrystals: chemistry, self-assembly and applications," Chemical Reviews, vol. 110, no. 6, pp. 3479-3500, 2010.

[3] C. F. Castro-Guerrero, M. R. Diaz-Guillen, F. Delgado-Arroyo, A. Rodas-Grapain, and S. Godavarthi, "Purification of cellulose from rice husk for the synthesis of nanocellulose," in 2016 IEEE 16th International Conference on Nanotechnology (IEEE-NANO), Sendai, Japan, 2016IEEE.

[4] R. K. Mishra, A. Sabu, and S. K. Tiwari, "Materials chemistry and the futurist eco-friendly applications of nanocellulose: status and prospect," Journal of Saudi Chemical Society, vol. 22, no. 8, pp. 949-978, 2018.

[5] G. A. Eyebe, B. Bideau, E. Loranger, N. Boubekeur, and F. Domingue, "Novel TOCN/PVOH dielectric composite sheets with low ecological footprint for microwave humidity sensing," IEEE Sensors Letters, vol. 2, no. 4, pp. 1-4, 2018.

[6] T. Ramu and H. Nagamani, "Alumina and silica based epoxy nanocomposites for electrical insulation," IEEE Transactions on Dielectrics and Electrical Insulation, vol. 21, no. 1, pp. 236-243, 2014.

[7] Y. J. Kang, S. J. Chun, S. S. Lee et al., “All-solid-state flexible supercapacitors fabricated with bacterial nanocellulose papers, carbon nanotubes, and triblock-copolymer ion gels," ACS Nano, vol. 6, no. 7, pp. 6400-6406, 2012.

[8] J. R. Nair, F. Bella, N. Angulakshmi, A. M. Stephan, and C. Gerbaldi, "Nanocellulose-laden composite polymer electrolytes for high performing lithium-sulphur batteries," Energy Storage Materials, vol. 3, pp. 69-76, 2016.

[9] D. Le Bras, M. Strømme, and A. Mihranyan, "Characterization of dielectric properties of nanocellulose from wood and algae for electrical insulator applications," Journal of Physical Chemistry B, vol. 119, no. 18, pp. 5911-5917, 2015.

[10] T. Okano and A. Sarko, "Mercerization of cellulose. I. X-ray diffraction evidence for intermediate structures," Journal of Applied Polymer Science, vol. 29, no. 12, pp. 4175-4182, 1984.

[11] C. F. Castro-Guerrero, A. B. Morales-Cepeda, M. R. DiazGuillen, F. Delgado-Arroyo, and F. A. Lopez-Gonzalez, Selfordered cellulose nanocrystals and microscopic investigations, Materials Science-Poland, in press, 2019.

[12] R. J. Samuels, "Solid-state characterization of the structure and deformation behavior of water-soluble hydroxypropylcellulose," Journal of Polymer Science Part A-2: Polymer Physics, vol. 7, no. 7, pp. 1197-1258, 1969.
[13] D. Gray, "Recent advances in chiral nematic structure and iridescent color of cellulose nanocrystal films," Nanomaterials, vol. 6, no. 11, p. 213, 2016.

[14] C. A. Castillo-Miranda, A. Beatriz Morales-Cepeda, C. F. Castro-Guerrero et al., "Deposition of Ibuprofen Crystals on Hydroxypropyl Cellulose/Polyacrylamide Gel: Experimental and Mathematic Modeling Releasing," International Journal of Polymer Science, vol. 2016, Article ID 2634104, 8 pages, 2016.

[15] C. A. Castillo-Miranda, C. F. Castro-Guerrero, H. A. VelascoOcejo et al., "Acetylsalicylic acid (ASA) on hydroxyethylcellulose/polyacrylamide gel (HEC/PAAm) as a proposal for a dermatological compress: mathematical modeling of ASA release kinetics," International Journal of Polymer Science, vol. 2019, Article ID 4597641, 10 pages, 2019.

[16] S. Evershed, "The characteristics of insulation resistance," Journal of the Institution of Electrical Engineers, vol. 52, no. 224, pp. 51-73, 1913.

[17] M. Roman and D. G. Gray, "Parabolic focal conics in selfassembled solid films of cellulose nanocrystals," Langmuir, vol. 21, no. 12, pp. 5555-5561, 2005.

[18] J.-F. Revol, H. Bradford, J. Giasson, R. H. Marchessault, and D. G. Gray, "Helicoidal self-ordering of cellulose microfibrils in aqueous suspension," International Journal of Biological Macromolecules, vol. 14, no. 3, pp. 170-172, 1992.

[19] D. Gray and X. Mu, "Chiral nematic structure of cellulose nanocrystal suspensions and films; polarized light and atomic force microscopy," Materials, vol. 8, no. 11, pp. 7873-7888, 2015.

[20] S. Shrestha, J. A. Diaz, S. Ghanbari, and J. P. Youngblood, "Hygroscopic swelling determination of cellulose nanocrystal (CNC) films by polarized light microscopy digital image correlation," Biomacromolecules, vol. 18, no. 5, pp. 1482-1490, 2017.

[21] G. Picard, D. Simon, Y. Kadiri, J. D. LeBreux, and F. Ghozayel, "Cellulose nanocrystal iridescence: a new model," Langmuir, vol. 28, no. 41, pp. 14799-14807, 2012.

[22] S. Maiti, J. Jayaramudu, K. Das et al., "Preparation and characterization of nano-cellulose with new shape from different precursor," Carbohydrate Polymers, vol. 98, no. 1, pp. 562-567, 2013.

[23] F. Shafizadeh and A. G. W. Bradbury, "Thermal degradation of cellulose in air and nitrogen at low temperatures," Journal of Applied Polymer Science, vol. 23, no. 5, pp. 1431-1442, 1979.

[24] C. Castro, A. Morales, A. Koschella, and T. Heinze, "Phase behaviour of hydroxypropyl cellulose/polyacrylamide gels," Macromolecular Symposia, vol. 296, no. 1, pp. 429-435, 2010.

[25] S. Maduskar, V. Maliekkal, M. Neurock, and P. J. Dauenhauer, "On the yield of levoglucosan from cellulose pyrolysis," ACS Sustainable Chemistry and Engineering, vol. 6, no. 5, pp. 7017-7025, 2018.

[26] A. Delvallée, N. Feltin, S. Ducourtieux, M. Trabelsi, and J. F. Hochepied, "Direct comparison of AFM and SEM measurements on the same set of nanoparticles," Measurement Science and Technology, vol. 26, no. 8, 2015.

[27] E. D. Cranston and D. G. Gray, "Morphological and optical characterization of polyelectrolyte multilayers incorporating nanocrystalline cellulose," Biomacromolecules, vol. 7, no. 9, pp. 2522-2530, 2006. 
[28] J. Majoinen, E. Kontturi, O. Ikkala, and D. G. Gray, “SEM imaging of chiral nematic films cast from cellulose nanocrystal suspensions," Cellulose, vol. 19, no. 5, pp. 1599-1605, 2012.

[29] https://www.doeeet.com/content/uncategorized/types-ofplastic-film-capacitor-dielectrics/.

[30] L. Caliari, P. Bettacchi, E. Boni et al., "KEMET film capacitors for high temperature, high voltage and high current," in CARTS International 2013 Proceedings, Houston, TX, 2013 Electronic Components Industry Association.

[31] J. Gamby, P. L. Taberna, P. Simon, J. F. Fauvarque, and M. Chesneau, "Studies and characterisations of various activated carbons used for carbon/carbon supercapacitors," Journal of Power Sources, vol. 101, no. 1, pp. 109-116, 2001.

[32] M. Natalia, Y. N. Sudhakar, and M. Selvakumar, "Activated carbon derived from natural sources and electrochemical capacitance of double layer capacitor," Indian Journal of Chemical Technology, vol. 20, no. 6, pp. 392-399, 2013.

[33] S. Araya, S. Andreasen, and S. Kær, "Experimental characterization of the poisoning effects of methanol-based reformate impurities on a PBI-based high temperature PEM fuel cell," Energies, vol. 5, no. 11, pp. 4251-4267, 2012.

[34] J. Sun and Y. Liu, "Unique constant phase element behavior of the electrolyte-graphene interface," Nanomaterials, vol. 9, no. 7, p. 923, 2019.

[35] I. Ahamad, R. Prasad, and M. A. Quraishi, "Adsorption and inhibitive properties of some new Mannich bases of Isatin derivatives on corrosion of mild steel in acidic media," Corrosion Science, vol. 52, no. 4, pp. 1472-1481, 2010.

[36] U. León-Silva, M. E. Nicho, and H. Hu, "Comparative study of optical kinetics in single and dual poly3-methylthiophenebased solid electrochromic devices," Journal of Solid State Electrochemistry, vol. 12, no. 1, pp. 71-80, 2007.

[37] C.-X. Liu, J. Chen, C. F. Zhang, H. H. Zhou, and G. Y. Han, "Facile preparation of binder-free electrode for electrochemical capacitors based on reduced graphene oxide composite film," Journal of Electroanalytical Chemistry, vol. 847, 2019.

[38] A. Virya and K. Lian, "Lithium polyacrylate-polyacrylamide blend as polymer electrolytes for solid- state electrochemical capacitors," Electrochemistry Communications, vol. 97, pp. 7781, 2018.

[39] B. Andres, C. Dahlström, N. Blomquist, M. Norgren, and H. Olin, "Cellulose binders for electric double-layer capacitor electrodes: the influence of cellulose quality on electrical properties," Materials and Design, vol. 141, pp. 342-349, 2018.

[40] M. Hou, M. Xu, Y. Hu, and B. Li, "Nanocellulose incorporated graphene/polypyrrole film with a sandwich-like architecture for preparing flexible supercapacitor electrodes," Electrochimica Acta, vol. 313, pp. 245-254, 2019. 